\title{
Lifestyle counselling as secondary prevention in patients with minor stroke and transient ischemic attack: study protocol for a randomized controlled pilot study
}

\author{
Jacob Liljehult 1,2,3* (i), Stig Molsted ${ }^{4}$, Tom Møller ${ }^{2,5}$, Dorthe Overgaard ${ }^{3}$, Lis Adamsen ${ }^{2,5}$, Mary Jarden ${ }^{5,6}$ and
} Thomas Christensen ${ }^{1,6}$

\begin{abstract}
Background: Most patients with minor stroke or transient ischemic attack (TIA) are discharged with little or no specialised follow-up. Nonetheless, these patients have a high prevalence of cognitive impairments and a considerable risk of recurrent stroke. Smoking cessation, physical activity, and adherence to antihypertensive and antithrombotic medication are highly recommended in patients with minor stroke and TIA. Evidence suggests that simple encouragement to change lifestyle is ineffective. Behavioural interventions might therefore be needed to support patients in managing their own health post-discharge.

Objectives: We aim to test the (1) feasibility of randomisation acceptance and an early initiated, client-centred lifestyle and behavioural intervention in a clinical setting, and (2) potential effect of the intervention on arterial blood pressure in patients with minor stroke or TIA and (3) explore the participants experience of barriers and facilitators for health behaviour after a stroke, including perceived needs and social support.
\end{abstract}

Methods: We will conduct a randomized controlled pilot trial: Eligible patients with acute minor stroke or TIA ( $n=$ 40) will be randomly allocated to either early initiated counselling with four weekly post-discharge follow-up sessions for 12 weeks or usual care. The primary outcome will be program feasibility and to discuss the relevance of arterial blood pressure as primary outcome after 12 weeks intervention. Selected participants will be invited to participate in semi-structured interviews, based on purposeful sampling, to evaluate the intervention and explore their experience of life after a stroke. The interviews will be analysed using a five-step thematic analysis approach.

(Continued on next page)

\footnotetext{
* Correspondence: jlil0004@regionh.dk

'Department of Neurology, Nordsjællands Hospital, Dyrehavevej 29, DK-3400

Hillerød, Denmark

${ }^{2}$ Department 9701, The University Hospitals Centre for Health Research UCSF,

Copenhagen University Hospital (Rigshospitalet), Blegdamsvej 9, DK-2100

Copenhagen, Denmark

Full list of author information is available at the end of the article
}

C C The Author(s). 2020 Open Access This article is licensed under a Creative Commons Attribution 4.0 International License, which permits use, sharing, adaptation, distribution and reproduction in any medium or format, as long as you give appropriate credit to the original author(s) and the source, provide a link to the Creative Commons licence, and indicate if changes were made. The images or other third party material in this article are included in the article's Creative Commons licence, unless indicated otherwise in a credit line to the material. If material is not included in the article's Creative Commons licence and your intended use is not permitted by statutory regulation or exceeds the permitted use, you will need to obtain permission directly from the copyright holder. To view a copy of this licence, visit http://creativecommons.org/licenses/by/4.0/ The Creative Commons Public Domain Dedication waiver (http://creativecommons.org/publicdomain/zero/1.0/) applies to the data made available in this article, unless otherwise stated in a credit line to the data. 
(Continued from previous page)

Discussion: The study will provide evidence of the feasibility and potential effect of early initiated counselling on cardiovascular risk factors in patients with minor stroke and TIA. Qualitative interviews will contribute with a more nuanced understanding of the barriers and facilitators of health enhancing behaviour. Optimizing health behaviour counselling and providing formal support to the patients' post-discharge may ease the transition and help more patients adhere to lifestyle and medication recommendations.

Trial registration: ClinicalTrial.gov, NCT03648957

Keywords: Stroke, Transient ischemic attack, Smoking, Exercise, Physical activity, Adherence, Early rehabilitation, Health counselling

\section{Background}

Stroke is a significant cause of morbidity, mortality, and disability in both western countries and globally [1]. In Denmark, the incidence rate is $3 / 1000$ per year, with a mortality rate of $10 \%$ in the first month [2]. More than half of patients admitted with a stroke or transient ischemic attack (TIA) only have mild neurological symptoms and are often discharged after 3-5 days of hospital admission. In spite of this, there is evidence that the patients after discharge often experience cognitive and communicative impairments such as difficulties with everyday activities, memory, fatigue, reading and participating in conversations [3]. This indicates that patients with recent minor stroke or TIA need more support than previously assumed.

One in four patients admitted with acute stroke has previously suffered a stroke or TIA. The risk is greatest shortly after the incident stroke; and within the first year the recurrence rate is $12-13 \%$ and subsequently plateauing at $5-6 \%$ per year [4]. Recurrent stroke is an independent risk factor for loss of function, institutionalization and death [5].

In the past decades, there has been an increasing focus on health behaviour in relation to the prevention of vascular diseases [6]. Arterial hypertension is the most important and prevalent risk factor for stroke and TIA (odds ratio (OR) 2.64 [2.26-3.08]; Population Attributable Risk (PAR) 34.6\%). However, other lifestyle factors, such as smoking (OR 2.09 [1.75-2.51]; PAR 18.9\%), physical inactivity (OR 1.45 [1.11-1.89]; PAR 28.5\%), abdominal obesity (OR 1.42 [1.18-1.71]; PAR 26.5\%), and unhealthy eating habits (OR 1.35 [1.12-1.61]; PAR 18.8\%) contribute to the risk of stroke [7].

Preventive medication is of utmost importance for secondary and tertiary prevention in patients with stroke and TIA. The majority of patients are treated with antithrombotic drugs or drugs to reduce blood pressure or blood lipids [8]. However, several studies have found that a substantial part of the patients discontinue the drug treatment overtime $[9,10]$.

The harmful effects derived from lifestyle factors, and the importance of adherence to preventive medication in relation to stroke is well-documented $[10,11]$. But there is still insufficient knowledge regarding how to most effectively communicate this to the patients and how to support the patient in making suitable choices to prevent recurrent strokes and progression of the disease. The results from previous studies have been contradictory. It is difficult to identify the most effective components and recommend specific interventions or components of interventions for implementation in clinical practice. Lawrence et al. [12] found in a systematic review of 20 randomized controlled trials that multimodal behavioural interventions had a beneficial effect on blood pressure in patients with stroke. Deijle et al. [13] found that lifestyle interventions had a greater effect if the intervention contained elements of physical activity. Lager et al. [14] on the contrary found no significant reduction in cardiovascular events in a systematic review of 26 randomized controlled trials of lifestyle intervention for stroke patients.

The time of inclusion in previous stroke/lifestyle intervention studies varied from a few days after stroke onset $[15,16]$ to several years [17]. The timing of the intervention might affect the patient's motivation and ability to adhere to the intervention, which will ultimately have an effect on the outcome. In other patient groups, it has been hypothesised that the time just after the diagnosis constitutes a certain window of opportunity; i.e. a limited period of time in which the patient is particularly receptive to information and behavioural changes [18].

The hypothesis of the present study is that early client-centred patient counselling with repeated followup sessions after discharge can reduce the blood pressure through smoking cessation, physical activity, and improved adherence to preventive medication in patients with minor stroke or TIA compared to simple encouragement to lifestyle change. The overall purpose of our research is to develop effective and clinically feasible interventions to support patients with minor stroke and TIA in engaging in health enhancing behaviour and ultimately prevent recurrent strokes.

Little research has explored how old age and cognitive impairments of this patient group affects their ability, readiness and willingness to participate in early initiated 
health behavioural interventions or if there are particular circumstances both during admission and post-discharge that should be taken into account when designing this type of research study.

The aims of this study are the following:

1) to evaluate the feasibility of a client-centred patient counselling intervention focused on smoking cessation, physical activity, and adherence to preventive medication in patients with minor stroke or TIA

2) to test the potential effects of the intervention on blood pressure and other cardiovascular risk factors in patients with minor stroke or TIA, and estimate means and standard deviations for subsequent sample-size calculations

3) to explore and evaluate the perceived needs and experience of social support of the participants in relation to health behavioural changes

\section{Methods}

The study will consist of a parallel group randomized controlled feasibility trial and a qualitative interview study. Participants will be randomly allocated to either an individual face-to-face counselling intervention, with follow-up sessions post-discharge at 4 week intervals for 12 weeks, or usual care. Semi-structured qualitative interviews will be conducted with selected participants after the last follow-up.

\section{Setting and participants}

The target population is hospitalised patients with recent minor stroke or TIA who are discharged home from the hospital.

We will include patients with acute minor stroke or TIA admitted to the Department of Neurology at Nordsjællands Hospital $(n=40)$. Nordsjællands Hospital is a university hospital with a catchment area of 310,000 citizens. All patients with stroke or TIA in the catchment area are admitted to the hospital and treatment is free of charge. The Department of Neurology has a specialized unit for patients with acute stroke and treatment is guided by a standardized patient pathway. Patients with minor stroke and TIA are generally hospitalised for observation for $72 \mathrm{~h}$.

\section{Study and recruitment procedures}

All new patients at the Department of Neurology will be screened for eligibility by the research investigator (JL). Patients are eligible if they are $\geq 18$ years old, are diagnosed with TIA (ICD-10 G45.9) or minor stroke (ICD10 I61, I63, I64; Scandinavian Stroke Scale 45-58), are discharged to their home, and can provide valid written consent. Diagnoses must be confirmed by a neurologist. Patients are excluded from the study if they have severe communication barriers, are not able to use a telephone, have severe disability prior to the stroke (WHO Performance Status $>2$; incapable of self-care and mobilised less than $50 \%$ of the day), have an active abuse of alcohol or narcotics or have a severe psychiatric illness (affective disease, dementia, schizophrenia, anxiety). Eligible patients will be invited to participate through verbal and written information regarding the study purpose and method by JL. All participants must give written informed consent before participation. Participant flow is summarised in Fig. 1.

\section{Randomization and group allocation}

Simple non-stratified 1:1 randomisation will be used to allocate participants into two trial arms (intervention or usual care) after baseline testing. Randomization will be conducted in the Research Electronic Data Capture (RedCap) software [19] using a computer generated randomization sequence. The randomization sequence will be generated by JL, then concealed in RedCap, and any changes can only be made by an external administrator. Participants will be automatically randomized after baseline testing and the allocation will be subsequently locked and cannot be altered. Participants will remain in the allocated arm for the entire intervention period.

\section{Intervention}

Participants in the intervention arm will receive usual care and a nurse-led targeted lifestyle counselling focusing on smoking cessation, everyday physical activity, and adherence to preventive medication. The initial counselling will be provided face-to-face by a research investigator before the participant is discharged from the hospital. The aim of the counselling will be to engage the participant to partake a healthy lifestyle and adhere to the preventive medication and to assist the participant in finding suitable strategies to achieve his/her goals. Participants will be encouraged to bring a relative or close friend to the initial counselling session, who can be a support for the participant after discharge. The counselling will be based on the 5A's approach (Ask/assess, Advice, Agree, Assist and Arrange, see Fig. 2) [20, 21]. Ask/assess. At baseline all participants will be asked about self-rated health, smoking habits, and physical activity, and body mass index, waist/hip-ratio and lung capacity will be assessed. During the counselling, the participants are asked to reflect on the following: the relevance of changing behaviour; how the specific behaviour affects their risk of disease; how they think changing behaviour could be beneficial as well as perceived barriers and facilitators of behavioural change. Perceived barriers and facilitators for changing behaviour can be both personal (e.g. attitudes towards changing behaviour, 


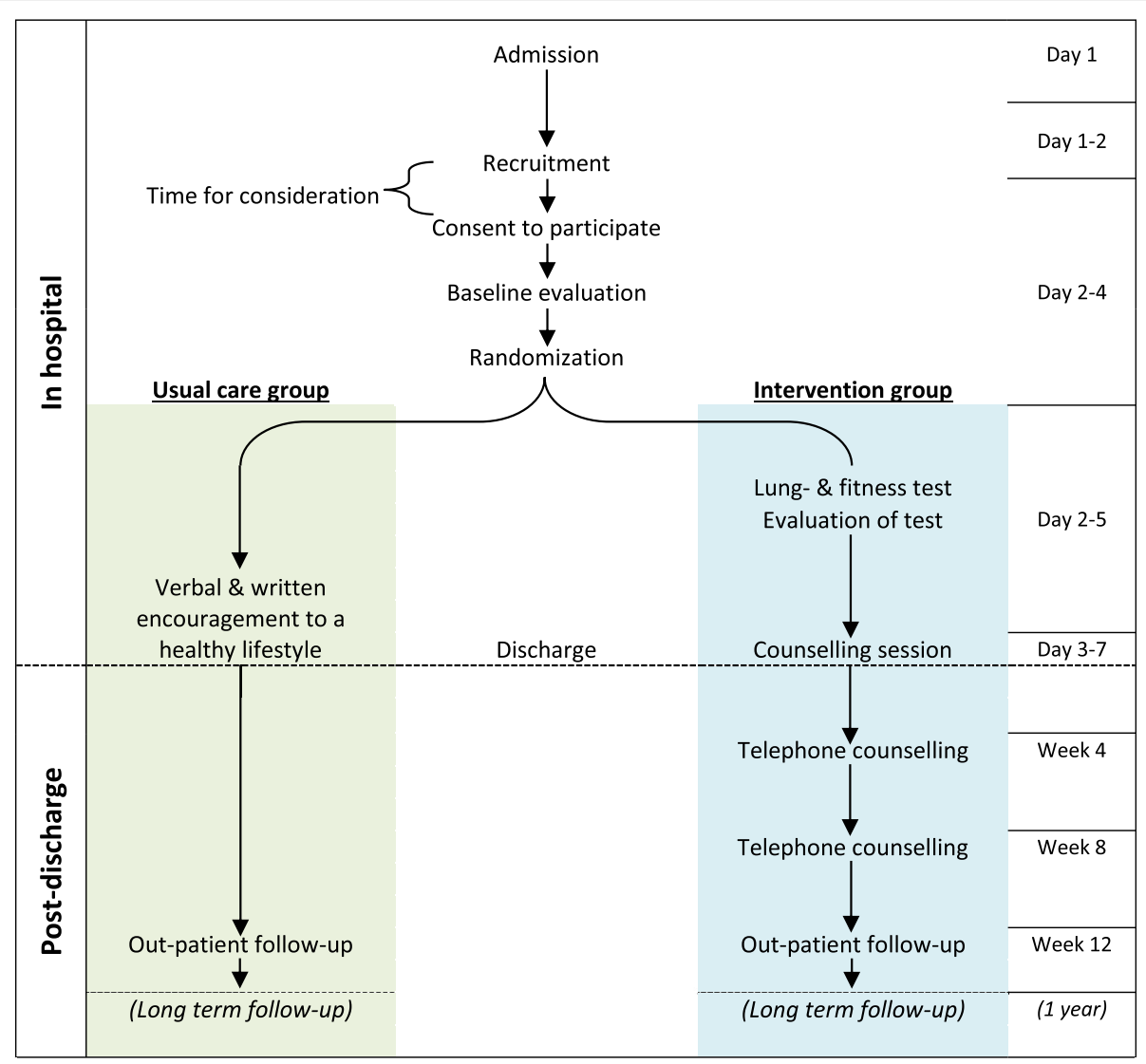

Fig. 1 Flowchart of recruitment, inclusion, allocation and follow-up of participants

attribution of risk and self-efficacy) and environmental (e.g. products, technology, surroundings, social support and services). The focus at this point in the counselling is not to find solutions, but to facilitate the participant's reflection on his or her health and health behaviour. Advise. The participant is given clear, specific and personal recommendations, including information on potential harms and benefits to their health and well-being. Agree. The participant is supported in setting goals. Assist. The investigator assists the participant in finding strategies to achieve the agreed-upon goals and helps the participant in acquiring the skills, confidence and social support within the participant's own environment to change behaviour. The strategies should be grounded in the previous reflections and customised to the needs, capacities, and preferences of the individual participant. Arrange. Follow-up sessions are arranged and, if necessary, the participant is referred to other relevant treatments.

Prior to of the initial counselling session a detailed assessment of the participant's lifestyle and physical condition will be conducted by JL (research investigator); including spirometry (FEV1/FVC measured using SpiroBank II) [22] and aerobic capacity (Åstrand-Rhyming Bike Test) [23].
Further counselling will be provided by telephone 4 and 8 weeks after discharge and will aim at maintaining motivation and adjusting goals and strategies if necessary. If the participant has not previously been motivated for changing behaviour, the willingness for change is re-assessed.

All parts of the intervention will be delivered by JL, who has extensive clinical experience as a nurse in the field of neurology.

After each counselling session, a written summary is included in the electronic patient record which is available to the participant via a personal log-in. The participant's relatives will be involved in the counselling at the participant's discretion to facilitate social support. Social support might be of particular importance in participants with cognitive impairments or limited ability to take in information.

The level of physical activity in the intervention arm will be monitored throughout the intervention period using an electronic activity tracker (Garmin VivoFit 4), which measures steps, intensity (light/moderate/vigorous activity) and estimated calories burned. The activity tracker should work as a reminder and motivator for everyday physical activity but will also provide feedback to support the post-discharge counselling. 


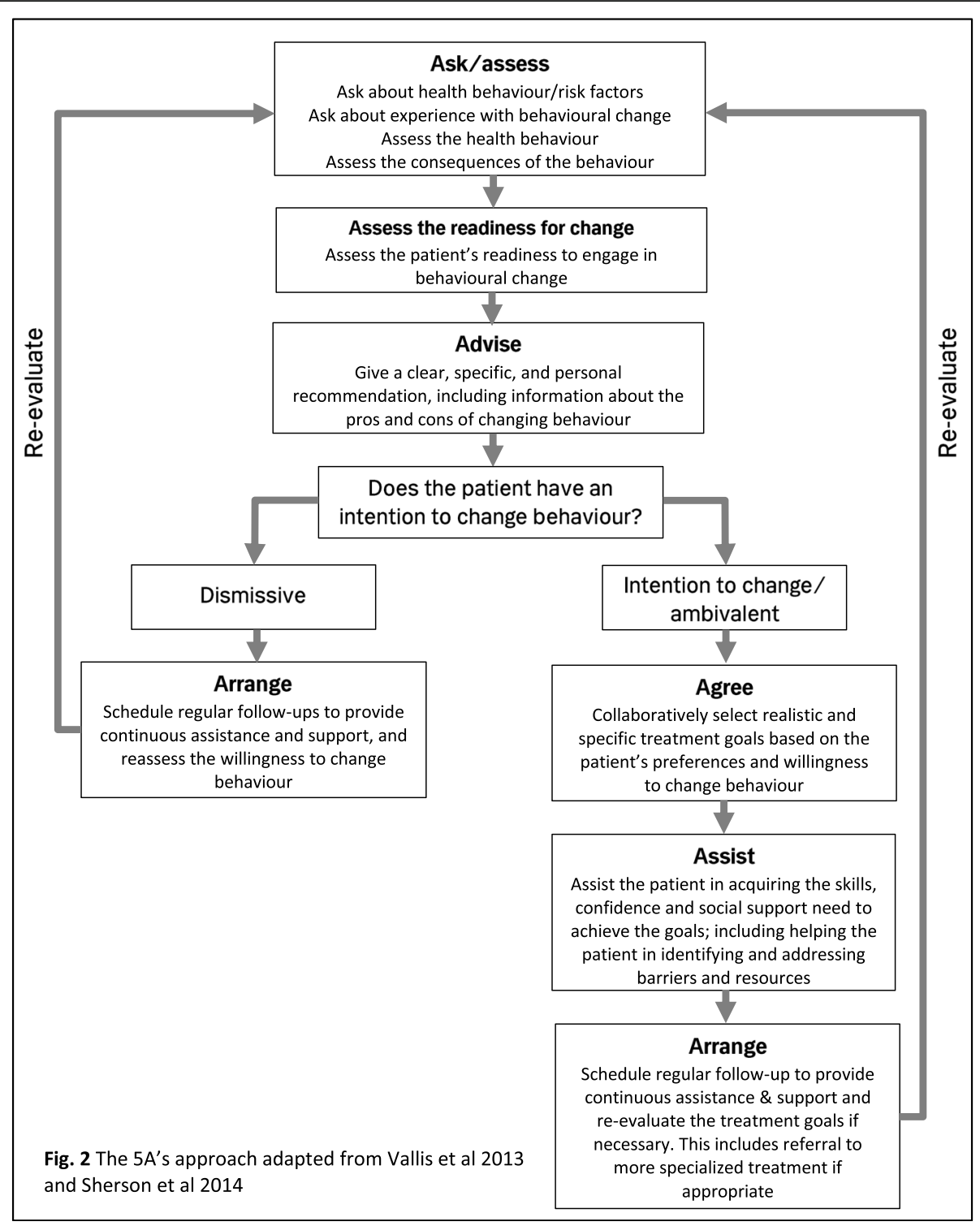

Fig. 2 The 5A's approach adapted from Vallis et al 2013 and Sherson et al 2014

\section{Usual care}

Participants in the control arm will receive standard care, which includes a review of prescribed medication, and both verbal and written encouragement of a healthy lifestyle (see Table 1) [24].

Table 1 Standard recommendations for a healthy lifestyle after stroke or TIA in Denmark [24]

- Smoking cessation is encouraged

- Regular physical activity is encouraged to the extent of the patient's

ability

- Patients with a substantial use of alcohol (> 7/14 units of alcohol per

week for women and men, respectively) should reduce the

consumption of alcohol or cease to use alcohol all together

- Patients are encouraged to eat a diet high in fruits, vegetables,

wholegrain products, and sea food; and to limit the intake of salt and saturated fats
Participants from both treatment arms will be reassessed in the hospital-based outpatient clinic 12 weeks after they are discharged from the hospital. The evaluation will include arterial blood pressure, smoking status, body mass index, waist/hip-ratio, and adherence to preventive medication (proportion of missed doses in the past 7 days) Fig 2.

\section{Study tests and assessments}

Baseline and outcome measures are summarised in Table 2.

\section{Baseline data}

After written consent has been obtained baseline data will be collected by the research investigator. Demographic 
Table 2 Baseline and outcome parameters

\begin{tabular}{|c|c|c|c|c|c|}
\hline Parameters & Methods & Baseline test & Discharge & 12-weeks follow-up & 1-year follow-up \\
\hline \multicolumn{6}{|l|}{ Baseline data } \\
\hline Demographic data & & $x$ & & & \\
\hline \multicolumn{6}{|c|}{$\begin{array}{l}\text { Age, gender, living conditions, education, performance } \\
\text { status [25] prior to stroke }\end{array}$} \\
\hline \multicolumn{6}{|l|}{ Health status } \\
\hline Stroke type & ICD-10 I61, 163, I64, G45.9 & $x$ & $x$ & & \\
\hline Stroke severity & Scandinavian Stroke Scale [26] & $x$ & & & \\
\hline Vital signs & Early warning score [27] & $x$ & & & \\
\hline Heart arrhythmia & Result of 48-72 h telemetry [28] & & $x$ & & \\
\hline Prior health problems & Charlson comorbidity index [29] & $x$ & & & \\
\hline Biochemistry & $\begin{array}{l}\text { Glucose, } \mathrm{HbA1c} \text {, cholesterol, } \mathrm{LDL} \text {, } \\
\text { VLDL, HDL, triglycerides [11] }\end{array}$ & & $x$ & & \\
\hline Prescribed medication & $\begin{array}{l}\text { Prescribed preventive medication } \\
\text { (Antihypertensives, antithrombotic, } \\
\text { anticoagulatives, NOAC, statins) }\end{array}$ & & $x$ & & \\
\hline \multicolumn{6}{|l|}{ Primary outcome } \\
\hline Eligibility rate & & $x$ & & & \\
\hline Study participation rate & & $x$ & & & \\
\hline Adherence to the program & & & & $x$ & \\
\hline Attrition rate & & & & $x$ & \\
\hline Satisfaction & & & & $x$ & \\
\hline Secondary outcome & & & & $x$ & \\
\hline Resting arterial blood pressure & $\begin{array}{l}\text { Average of two or more measurements } \\
\text { in sitting position after > } 10 \text { min resting } \\
\text { according to } 2017 \text { US Guidelines [30] }\end{array}$ & $x$ & & $x$ & \\
\hline \multicolumn{6}{|l|}{ Tertiary outcomes } \\
\hline Current smoking & $\begin{array}{l}\text { Self-reported tobacco smoking (daily, } \\
\text { weekly, rarely, has quit smoking, never } \\
\text { smoked) [31] }\end{array}$ & $x$ & & $x$ & \\
\hline Physical activity & $\begin{array}{l}\text { Self-reported participation in leisure time } \\
\text { physical activities (minutes per week of } \\
\text { light/moderate/strenuous) [32] }\end{array}$ & $x$ & & $x$ & \\
\hline Body composition & Body mass index, hip/waist ratio & $x$ & & $x$ & \\
\hline Fatigue & $\begin{array}{l}\text { Fatigue Assessment Scale [33]; 10-item } \\
\text { questionnaire }\end{array}$ & $x$ & & $x$ & \\
\hline Self-rated health & $\begin{array}{l}\text { Self-rated health current and in the last } \\
\text { year [31] }\end{array}$ & $x$ & & $x$ & \\
\hline Adherence to preventive medication & $\begin{array}{l}\text { Adherence to preventive medication } \\
\text { (Antihypertensives, antithrombotic, } \\
\text { anticoagulatives, NOAC, statins) the last } \\
7 \text { days prior to assessment }\end{array}$ & & & $x$ & \\
\hline Units of alcohol per week & Self-reported [31] & $x$ & & $x$ & \\
\hline Recurrent stroke/TIA & Patient medical report data & & & & $x$ \\
\hline Ischemic heart disease & Patient medical report data & & & & $x$ \\
\hline All-cause mortality & Danish Central Person Registry & & & & $x$ \\
\hline
\end{tabular}

$L D L$ low-density lipoprotein, $V L D L$ very low-density lipoprotein, $H D L$ high-density lipoprotein, NOAC novel oral anticoagulants

data will include age, gender, living arrangements, educational level and ECOG performance status [25] prior to stroke onset. Lifestyle factors will include self-rated health, smoking habits, alcohol consumption and anthropometric measures, assessed using standardised questions from the Danish National Health Survey Questionnaire [31]. The International Physical Activity Questionnaires short form (IPAQ-SF) [32] will be used to assess the level of physical 
activity prior to admission. Scandinavian Stroke Scale [26] will be used to assess stroke severity. Charlson Comorbidity Index [29] will be used to assess the burden of comorbidities. Early Warning Score [27] will be used to summarize vital signs. Serum lipids (total cholesterol, HDL, LDL, VLDL, triglycerides), random blood glucose and HbA1c are routinely collected on all patients [11].

\section{Outcome measures}

Measures of feasibility

The primary outcome measures are the following:

1) the eligibility rate (proportion of eligible patients compared to the total number of stroke patients)

2) the study participation rate (proportion of patients accepting participation in the study)

3) the degree of adherence to the program (proportion of attendance in follow-up sessions)

4) attrition (drop-out and withdrawal)

5) participant satisfaction with the intervention, using both quantitative (Likert scale) and qualitative (semi-structured interviews) measures, to guide the design of a future full-scaled randomized controlled trial

\section{Measure of potential effect}

The secondary outcome measure will be arterial blood pressure measured before and 12 weeks after the start of the intervention. Measurements will be performed in accordance with the American Heart Association guidelines [30]. To avoid extreme values, the blood pressure will be measured at least twice. If the two measurements of the systolic pressure are more than $5 \mathrm{mmHg}$ apart the measurement will be repeated. The average of the last two measurements will be used for analysis.

\section{Other outcome measures}

Tertiary outcomes after 12 weeks will include the following: self-reported smoking status, physical activity level (IPAQ-SF) [32], adherence to medication (number of missed/consumed doses in the past seven days), anthropometrics (body weight, waist/hip ratio) and Fatigue Assessment Scale [33].

\section{Long term outcome}

All participants will be followed up twelve months after the incident stroke/TIA through the national patient registry, which contains information on all patient admissions in Danish hospitals. The outcome measure will be recurrent strokes/TIA, myocardial infarcts, other cardiac admissions and death.

\section{Analysis plan Quantitative data}

Data will be entered into a RedCap database in real-time using electronic case report forms. Statistical analyses will be carried out using R 3.3.1/R Studio 0.99.

Recruitment, randomization, allocation and follow-up will be reported in a flow-chart stating the number of participants at each step. Characteristics of participants and non-participants (e.g. excluded, non-consenting or lost to follow-up) will be reported as detailed as possible according to CONSORT guidelines for feasibility studies [34].

Baseline data on participant demographics and characteristics will be presented in a descriptive table in accordance with the CONSORT guidelines.

The primary endpoint (12 weeks study retention in the two randomization groups) will be reported as absolute numbers and proportions with 95\% confidence intervals. The secondary endpoint (arterial blood pressure) will be reported as an absolute number for each group and estimate of the between group difference with $95 \%$ confidence intervals. Twelve months follow-up data will be reported as absolute numbers, absolute risk reduction and risk ratio (with 95\% confidence intervals) comparing the risks of a negative event (recurrent stoke, TIA, cardiovascular incident or death) in the two groups. An intention-to-treat approach will be employed in the analysis of all endpoints.

\section{Qualitative data}

Based on purposeful sampling [35] selected participants from both randomization groups will be invited to participate in in-depth semi-structured interviews. The participants are encouraged to invite a relative to participate in the interview. The sampling strategy will aim at achieving maximal variation concerning sex, time since discharge, and whether they have been successful in changing behaviour. The sample size will be based on achieving theoretical data saturation (expected $n=15$ 20). A semi-structured interview guide will be designed to give structure to the interviews.

The interviews will be digitally recorded and transcribed, and analysed using thematic analysis as described by Braun and Clarke [36]. The qualitative software system NVivo version 12 (QSR International) will be used to organize data and support the process of analysis.

\section{Approvals and registrations}

The study protocol has been approved by the Scientific Committee of the Capital Region (H-17040484) and the Danish Data Protection Agency (j.nr. VD-2018-306, I6552). The study protocol is registered at ClinicalTrials. gov (NCT03648957). 


\section{Discussion}

Most patients with TIA and minor stroke are discharged to their own home with little or no specialised followup. Previous studies have reported a high prevalence of cognitive impairments such as poor memory, fatigue and difficulty with reading and writing [3] as well as a high risk of stroke recurrence [37, 38]. The overall purpose of the current study is to develop an early initiated, clientcentred behavioural intervention to support patients with minor stroke and TIA in managing their own health post-discharge. The intervention is pragmatically integrated within the existing standard treatment and guidelines.

Self-management of health is complex and requires both knowledge, skills, and confidence [39]. Optimizing counselling and providing formal support from health professionals might help patients obtain a greater adherence to lifestyle recommendations and medication prescriptions.

The rationale behind early initiation of the intervention was an assumption that patients might be more susceptible to information and behavioural change close to the initial event. In studies of other patient populations, it has been hypothesized that the time close to the initial diagnosis constitutes a certain window of opportunity, i.e. a limited period of time where the patient is more receptive to information [18]. In previous studies of behavioural interventions in patients with stroke and TIA, it has been shown that the time of inclusion has varied from a few days to several years post-stroke [15-17]. This might be part of the reason why results have been inconsistent.

Part of the purpose of the pilot study will therefore be to examine whether the patients are willing and able to participate in this type of intervention in the early phase of their disease. We anticipate that some patients will not be ready to participate at this stage, either because they find it too overwhelming to participate or because they are unable to take in the amount of information needed to engage in behavioural change.

Furthermore, we will examine whether it is feasible to initiate and complete the counselling within the limited time of admission in an acute setting. Patients with minor stroke and TIA are generally hospitalized for 3-4 days ( $72 \mathrm{~h}$ of observation) and this time frame might prove to be difficult to identify and include relevant patients in the study and provide the intervention.

Smoking, physical activity, and adherence to preventive medication were chosen as the main focus of the counselling intervention as these factors have been shown to be both modifiable $[40,41]$ and, in our judgement, were the most likely to have an impact on the systolic blood pressure $[42,43]$.

Systolic blood pressure was chosen as the main measure of effect because it is the greatest risk factor of both ischemic and haemorrhagic stroke [7] and has been demonstrated to be modifiable in previous intervention studies [12].

The randomized controlled design will be used to test the feasibility and acceptability of the overall study methodology and the specific elements of the intervention. This includes testing whether the screening procedure is suitable for identifying relevant patients, inclusion and exclusion criteria has the right balance of sensitivity and specificity, the procedure of recruiting and attaining consent is suitable, and delivery of the intervention and follow-up is acceptable. Through this process, we will gain knowledge and experience which can guide further development of the study protocol [44].

The qualitative interviews with the study participants will be used to gain insights into the lived experience of life going home after hospitalisation for stroke, as well as their experience of managing their own health. This will give us a more profound understanding of the needs of patients who are discharged from the hospital and which type of support and counselling should be provided before and after the discharge.

Furthermore, the qualitative interviews will be used to evaluate the randomized controlled trial and the intervention. Even though this area of research is relatively new, previous studies have found that engaging patients and other stakeholders in the development of clinical studies might help the researcher in developing more meaningful and feasible methods and to identify more useful outcome measures [45]. The qualitative interviews might also contribute with a deeper and more nuanced understanding of the barriers and facilitators of health enhancing behaviour in patients with stroke. The needs of this particular group of patients might differ from other patient groups because of the combination of older age and cognitive impairments.

A key part of a pilot study is to evaluate the feasibility of conducting a full-sized trial in the future.

A potential impediment for conducting a full-sized trial of this type might be a restricted number of willing participants. To assess whether a full-sized trial is feasible, we must therefore (1) assess how many participants can realistically be recruited within a reasonable time period, and (2) analyse available data on non-participants and low adherence to find potential strategies to reduce non-participation.

\section{Limitations}

The present study will have some limitations. First off, we wish to evaluate the feasibility of the study design and the intervention, and the sample size of the study will therefore be limited increasing the risk of both type 1 and type 2 error. The sample size of 40 participants was not based on a formal sample size calculation, but rather on a pragmatic estimate of how many participants 
we would be possible to recruit within a reasonable timeframe, knowing full well that the trial would likely be under-powered for hypothesis testing. Though we assumed that 40 participants would be enough to reliable test all procedures of the trial even with a substantial rate of attrition.

The measurement of blood pressure will be done according to a standardized study manual to increase the validity and reliability. Self-reported data at baseline and follow-up will be collected using the same method in both treatment groups to minimize information bias.

Given the nature of the intervention the possibility of blinding is limited. Blinding of the assessors or participants is not possible in studies in which patient activation and counselling is part of the intervention. However, blinding in the analysis of the quantitative data will be possible.

\section{Ethical considerations}

The study will be conducted in accordance with the Helsinki Declaration [46] including respect for the participants' autonomy and right to informed consent. Participants will be informed that participation is voluntary, and that participation can be declined at any time and without explanation. Participant data will be kept confidential in accordance with guidelines from the Danish Data Protection Agency.

The inconveniences of participating will be minor, and we are convinced that the potential benefits will outweigh the drawbacks. Withdrawal symptoms associated with smoking cessation and muscle soreness associated with increased physical activity are to be expected. Generally, these will be both minor and transient [47, 48]. Participants will be encouraged to report any suspected adverse effects, including side effects to the medication as this might affect adherence. At all follow-up contacts (telephone and outpatient), the participants will be asked if they have experienced adverse effects or side effects to the medication. If potentially serious adverse effects are reported, a department physician will be consulted. All reports of adverse or side effects will be qualitatively evaluated and quantitatively compared between the trial arms.

The use of a control group as a comparison is necessary if we wish to find evidence of the potential hypothesized effect of the treatment and gain a better understanding of the variation in the outcome measures. It will also be necessary to test the feasibility of the randomization process prior to the design of a full scale randomized controlled trial. The treatment of the participants in the control group will at no point be inferior to the treatment of nonparticipants.

\section{Dissemination}

Results of the study will be published in international peerreviewed scientific journals and presented at national and international conferences. Results will be made public regardless of them being positive, negative, or inconclusive.

The principal investigator (JL) will draft all publications under the supervision of the other members of the project group. The order of authorship will be in order of contribution and has been agreed upon in the project group beforehand. The rights and responsibilities of the authors will be in accordance with The Danish Code of Conduct for Research Integrity [49] and the ICMJE recommendations [50].

\section{Trial status}

Recruitment has been completed in January 2020.

\section{Acknowledgements}

We wish to thank the Department of Neurology's head nurse Lene Hamberg, $\mathrm{RN}, \mathrm{MPO}$, for her collaboration in establishing the research program.

\section{Project organization}

The project was initiated as part of the CIRE neuro/psych research program in collaboration between Nordsjællands Hospital, Hillerød; Copenhagen

University College; and The University Hospitals Centre for Health Research UCSF, Copenhagen University Hospital (Rigshospitalet).

The instigators of the CIRE neuro/psych research program consist of researchers and managers from UCSF and Copenhagen University College, in collaboration with the UCSF steering committee consisting of

representatives of the hospital managers in Region Hovedstaden.

Progress of the project is presented to the members of the CIRE neuro/psych collaboration at bi-yearly meetings with representatives of all the involved parties. The UCSF steering committee has initiated the program but has no direct involvement in the design or implementation of the individual studies.

\section{Authors' contributions}

$J \mathrm{~L}$ is the principal investigator who will conduct the study and has drafted the article. The protocol was designed by $\mathrm{J}$ in collaboration with TC, TM, DO, SM, MJ and LA. TC will be the responsible neurologist. All authors have made a substantial contribution to the conception and design of the study protocol and all authors approved the final manuscript.

\section{Funding}

The pilot study has received funding from Nordsjællands Hospital, The University Hospital Centre for Health Research, Copenhagen University College through the CIRE neuro/psych research program, and extern funding from Anita \& Tage Therkelsens Fond and TrygFonden (ID 126993). The CIRE neuro/psych collaboration has been involved in the design of the study protocol and is acting as overseers of the execution of the study.

\section{Availability of data and materials}

Data used during the current study will be available from the corresponding author upon reasonable request.

\section{Ethics approval and consent to participate \\ Approved by the Danish Data Protection Agency (j.nr. VD-2018-306, I-6552) The protocol is in accordance with the rules of the Scientific Committee of the Capital Region, Denmark ( $\mathrm{H}-17040484)$. The study protocol is registered at ClinicalTrials.gov (NCT03648957). Registered 28 August 2018, https://clini- caltrials.gov/ct2/show/record/NCT03648957.}

\section{Consent for publication}

Not applicable

\section{Competing interests}

None

\section{Author details}

${ }^{1}$ Department of Neurology, Nordsjællands Hospital, Dyrehavevej 29, DK-3400

Hillerød, Denmark. ²Department 9701, The University Hospitals Centre for 
Health Research UCSF, Copenhagen University Hospital (Rigshospitalet), Blegdamsvej 9, DK-2100 Copenhagen, Denmark. ${ }^{3}$ Faculty of Health and Technology, Institute of Nursing and Nutrition, Copenhagen University College, Tagensvej 86, DK-2200 Copenhagen N, Denmark. ${ }^{4}$ Department of Clinical Research, Nordsjæellands Hospital, Dyrehavevej 29, DK-3400 Hillerød, Denmark. Institute of Public Health, University of Copenhagen, Øster Farimagsgade 5, DK-1353 Copenhagen K, Denmark. ${ }^{6}$ Department of Clinical Medicine, University of Copenhagen, Blegdamsvej 3, DK-2200 Copenhagen N, Denmark

\section{Received: 29 March 2019 Accepted: 11 March 2020} Published online: 25 March 2020

\section{References}

1. Feigin $\mathrm{VL}$, Forouzanfar $\mathrm{MH}$, Krishnamurthi $\mathrm{R}$, et al. Global and regional burden of stroke during 1990-2010: findings from the Global Burden of Disease Study 2010. Lancet (London, England). 2014;383:245-54.

2. Ingeman A (ed). Dansk Apopleksiregister - Årsrapport 2013. 2014.

3. Fens M, van Heugten CM, Beusmans GHMI, et al. Not as transient: patients with transient ischaemic attack or minor stroke experience cognitive and communication problems; an exploratory study. Eur J Gen Pract. 2013;19:11-6.

4. Olsen TS. Chapter 21 Stroke recurrence and prognosis after stroke. In: Handbook of clinical neurology. Edinburgh; New York: Elsevier, pp. 407-421.

5. Hankey GJ, Jamrozik K, Broadhurst RJ, et al. Long-term disability after firstever stroke and related prognostic factors in the Perth Community Stroke Study, 1989-1990. Stroke. 2002;33:1034-40.

6. World Health Organization. The World Health Report 2002: reducing risks to health, promoting healthy life. 1st ed. Geneva: WHO, http://apps.who.int/ iris/bitstream/handle/10665/42510/WHR_2002.pdf; 2003.

7. O'Donnell MJ, Xavier D, Liu L, et al. Risk factors for ischaemic and intracerebral haemorrhagic stroke in 22 countries (the INTERSTROKE study): a case-control study. Lancet (London, England). 2010;376:112-23.

8. European Stroke Organisation (ESO) Executive Committee, ESO Writing Committee. Guidelines for management of ischaemic stroke and transient ischaemic attack 2008. Cerebrovasc Dis. 2008;25:457-507.

9. Wessol JL, Russell CL, Cheng AL. A systematic review of randomized controlled trials of medication adherence interventions in adult stroke survivors. J Neurosci Nurs. 2017:49:120-33.

10. Glader E-L, Sjölander M, Eriksson M, et al. Persistent use of secondary preventive drugs declines rapidly during the first 2 years after stroke. Stroke. 2010:41:397-401.

11. Kernan WN, Ovbiagele B, Black HR, et al. Guidelines for the prevention of stroke in patients with stroke and transient ischemic attack: a guideline for healthcare professionals from the American Heart Association/American Stroke Association. Stroke. 2014;45:2160-236.

12. Lawrence M, Pringle J, Kerr S, et al. Multimodal secondary prevention behavioral interventions for TIA and stroke: a systematic review and metaanalysis. PLoS One. 2015;10:e0120902.

13. Deijle IA, Van Schaik SM, Van Wegen EEH, et al. Lifestyle interventions to prevent cardiovascular events after stroke and transient ischemic attack: systematic review and meta-analysis. Stroke. 2017:48:174-9.

14. Lager KE, Mistri AK, Khunti $K$, et al. Interventions for improving modifiable risk factor control in the secondary prevention of stroke. Cochrane database Syst Rev 2014; (5):CD0091: CD009103.

15. Joubert J, Reid C, Barton D, et al. Integrated care improves risk-factor modification after stroke: initial results of the Integrated Care for the Reduction of Secondary Stroke model. J Neurol Neurosurg Psychiatry. 2009; 80:279-84.

16. Boss HM, Van Schaik SM, Deijle IA, et al. Safety and feasibility of post-stroke care and exercise after minor ischemic stroke or transient ischemic attack: MotiveS \& MovelT. NeuroRehabilitation. 2014;34:401-7.

17. English C, Healy GN, Olds T, et al. Reducing sitting time after stroke: a phase II safety and feasibility randomized controlled trial. Arch Phys Med Rehabil. 2016:97:273-80

18. Møller T, Lillelund C, Andersen C, et al. At cancer diagnosis: a 'window of opportunity' for behavioural change towards physical activity. A randomised feasibility study in patients with colon and breast cancer. BMJ Open. 2013;3: e003556.

19. Vanderbilt. Research Electronic Data Capture - About, https://projectredcap. org/about/ (2017, accessed 9 July 2018).
20. Sherson EA, Yakes Jimenez E, Katalanos N. A review of the use of the 5 A's model for weight loss counselling: differences between physician practice and patient demand. Fam Pract. 2014;31:389-98.

21. Vallis M, Piccinini-Vallis $\mathrm{H}$, Sharma AM, et al. Modified 5 As: minimal intervention for obesity counseling in primary care. Can Fam Physician. 2013;59:27-31.

22. Miller MR, Hankinson J, Brusasco V, et al. Standardisation of spirometry. Eur Respir J. 2005:26:319-38.

23. Lennon OC, Denis RS, Grace N, et al. Feasibility, criterion validity and retest reliability of exercise testing using the Astrand-Rhyming test protocol with an adaptive ergometer in stroke patients. Disabil Rehabil. 2012;34:1149-56.

24. Dansk Selskab for Apopleksi. Referenceprogram for behandling af patienter med apopleksi. 3. København: Dansk selskab for apopleksi; 2012.

25. Suh SY, Leblanc TW, Shelby RA, et al. Longitudinal patient-reported performance status assessment in the cancer clinic is feasible and prognostic. J Oncol Pract. 2011;7:374-81.

26. Lindenstrøm E, Boysen G, Waage Christiansen L, et al. Reliability of Scandinavian Neurological Stroke Scale. Cerebrovasc Dis. 1991;1:103-7.

27. Liljehult J, Christensen T. Early warning score predicts acute mortality in stroke patients. Acta Neurol Scand. 2016;133:261-7.

28. Grond $M$, Jauss M, Hamann $G$, et al. Improved detection of silent atrial fibrillation using 72-hour Holter ECG in patients with ischemic stroke: a prospective multicenter cohort study. Stroke. 2013;44:3357-64.

29. Sundararajan V, Henderson T, Perry $C$, et al. New ICD-10 version of the Charlson comorbidity index predicted in-hospital mortality. J Clin Epidemiol. 2004;57:1288-94.

30. Stergiou G, Palatini $P, A s m a r ~ R$, et al. Blood pressure measurement and hypertension diagnosis in the 2017 US Guidelines. Hypertension. 2018;71: 963-5.

31. Christensen Al, Ekholm O, Glümer C, et al. The Danish National Health Survey 2010. Study design and respondent characteristics. Scand J Public Health. 2012:40:391-7.

32. Ekelund U, Sepp H, Brage S, et al. Criterion-related validity of the last 7-day, short form of the International Physical Activity Questionnaire in Swedish adults. Public Health Nutr. 2006;9:258-65.

33. De Vries J, Michielsen $H$, Van Heck GL, et al. Measuring fatigue in sarcoidosis: the Fatigue Assessment Scale (FAS). Br J Health Psychol. 2004;9: 279-91.

34. Schulz KF, Altman DG, Moher D, et al. CONSORT 2010 Statement: updated guidelines for reporting parallel group randomised trials. Trials. 2010;11:32.

35. Malterud K. Kvalitative forskningsmetoder for medisin og helsefag. 4. utgave. Oslo: Universitetsforlaget; 2017.

36. Braun V, Clarke V. Using thematic analysis in psychology. Qual Res Psychol. 2006:3:77-101.

37. Ois A, Gomis M, Rodríguez-Campello A, et al. Factors associated with a high risk of recurrence in patients with transient ischemic attack or minor stroke. Stroke 2008:39:1717-21.

38. Zhang C, Zhao X, Wang C, et al. Prediction factors of recurrent ischemic events in one year after minor stroke. PLoS One. 2015;10:e0120105.

39. Hibbard JH, Greene J. What the evidence shows about patient activation: better health outcomes and care experiences; fewer data on costs. Health Aff (Millwood). 2013;32:207-14.

40. Rice $V H$, Heath L, Livingstone-Banks J, et al. Nursing interventions for smoking cessation. Cochrane database Syst Rev. 2017;12:CD001188.

41. Conn VS, Ruppar TM. Medication adherence outcomes of 771 intervention trials: systematic review and meta-analysis. Prev Med (Baltim). 2017:99:269-76.

42. Ferdinand KC, Nasser SA. Management of essential hypertension. Cardiol Clin. 2017;35:231-46

43. Minami J, Ishimitsu T, Matsuoka H. Effects of smoking cessation on blood pressure and heart rate variability in habitual smokers. Hypertens (Dallas, Tex 1979). 1999;33:586-90.

44. Leon AC, Davis LL, Kraemer HC. The role and interpretation of pilot studies in clinical research. J Psychiatr Res. 2011;45:626-9.

45. Domecq JP, Prutsky G, Elraiyah $T$, et al. Patient engagement in research: a systematic review. BMC Health Serv Res. 2014;14:89.

46. World Medical Association. World Medical Association Declaration of Helsinki: ethical principles for medical research involving human subjects. JAMA. 2013:310:2191-4.

47. Hughes JR. Effects of abstinence from tobacco: etiology, animal models, epidemiology, and significance: a subjective review. Nicotine Tob Res. 2007; 9:329-39. 
48. Goodrich DE, Larkin AR, Lowery JC, et al. Adverse events among high-risk participants in a home-based walking study: a descriptive study. Int J Behav Nutr Phys Act. 2007:4:20.

49. Ministry of Higher Education and Science. Danish code of conduct for research integrity. Kbh.: Ministry of Higher Education and Science, https:// ufm.dk/publikationer/2014/filer-2014/the-danish-code-of-conduct-forresearch-integrity.pdf (2014).

50. International Committee of Medical Journal Editors. Recommendations for the conduct, reporting, editing, and publication of scholarly work in medical journals. December, http://www.icmje.org/icmje-recommendations. pdf (2017, accessed 7 November 2018).

\section{Publisher's Note}

Springer Nature remains neutral with regard to jurisdictional claims in published maps and institutional affiliations.

Ready to submit your research? Choose BMC and benefit from:

- fast, convenient online submission

- thorough peer review by experienced researchers in your field

- rapid publication on acceptance

- support for research data, including large and complex data types

- gold Open Access which fosters wider collaboration and increased citations

- maximum visibility for your research: over $100 \mathrm{M}$ website views per year

At $\mathrm{BMC}$, research is always in progress.

Learn more biomedcentral.com/submissions 